\title{
Direct Evidence for Biphasic cAMP Responsive Element-Binding Protein Phosphorylation during Long-Term Potentiation in the Rat Dentate Gyrus In Vivo
}

\author{
Stefan Schulz, Helge Siemer, Manfred Krug, and Volker Höllt \\ Department of Pharmacology and Toxicology, Otto-von-Guericke University, 39120 Magdeburg, Germany
}

Phosphorylation of the transcription factor cAMP responsive element-binding protein (CREB) is thought to play a key role in synaptic plasticity and long-term memory. However, direct evidence for CREB phosphorylation during hippocampal longterm potentiation (LTP) in vivo is sparse. Here, we show that, in the intact animal, CREB is rapidly phosphorylated in response to high-frequency stimulation but not low-frequency stimulation of the perforant pathway. CREB phosphorylation occurred in a biphasic manner, with a first peak at 30 min and a second long-lasting peak beginning $2 \mathrm{hr}$ after tetanic stimulation and lasting for at least $24 \mathrm{hr}$. Only stimuli that generated nondecremental LTP promoted a sustained hyperphosphorylation of CREB but not stimuli that produced decremental LTP. CREB phosphorylation was specifically triggered in the dentate gyrus, as well as the CA1, but not the CA3, hippocampal region. Pretreatment with the NMDA receptor antagonist (+)-5-methyl10,11-dihydro-5H-dibenzo [a,d] cyclohepten-5,10-imine maleate completely prevented activation of CREB. Together, we have resolved the spatial and temporal dynamics of CREB phosphorylation during hippocampal LTP, showing that the transcription factor CREB is specifically recruited at two distinct time points in some forms of hippocampal synaptic plasticity in vivo.

Key words: CREB; phosphorylation; LTP; hippocampus; antibodies; immunocytochemistry
Hippocampal long-term potentiation (LTP) is an activitydependent strengthening of synaptic efficacy that can last for days or weeks in intact animals and may be a useful model for studying learning-induced changes in synaptic activity (Bliss and Collingridge, 1993). In an interesting parallel to learning and memory, LTP has at least two functionally and mechanistically distinct phases. Whereas the induction phase (1-3 hr) is generally believed to be mediated via activation of existing proteins, i.e., by phosphorylation or isoprenylation (Bailey et al., 1996; Matthies et al., 1997a,b), maintenance of LTP requires de novo RNA transcription and protein synthesis (Krug et al., 1984; Otani and Ben Ari, 1993).

Substantial evidence has evolved indicating that the cAMPresponsive element-binding protein (CREB) plays a central and highly conserved role in the formation of long-term memory (for review, see Frank and Greenberg, 1994; Stevens, 1994; Bailey et al., 1996). Long-term facilitation of sensory neurons in Aplysia is blocked by injection of CRE oligonucleotides into the presynaptic nucleus (Dash et al., 1990). Furthermore, relief of repression of the inhibitory CREB2 isoform converted transient facilitation in Aplysia into long-term facilitation (Bartsch et al., 1995, 1998). In transgenic Drosophila, long-term memory disappears or is augmented after induction of repressor or activator forms of CREB, whereas short-term memory appears to be intact (Yin et al., 1994, 1995). Mutant mice lacking CREB isoforms $\alpha$ and $\delta$ have defi-

\footnotetext{
Received Dec. 22, 1998; revised April 20, 1999; accepted April 21, 1999.

This work was supported by Deutsche Forschungsgemeinschaft Grant SCHU 924/4-1 to S.S., Kultusministerium des Landes Sachsen/Anhalt Grant 1908A/0025 to S.S., and Sonderforschungsbereich 426 Grant $125572-2$ to M.K. We thank Maria Wagner and Dana Wiborny for excellent technical assistance.

Correspondence should be addressed to Volker Höllt, Department of Pharmacology and Toxicology, Otto-von-Guericke University, Leipziger Strasse 44, 39120 Magdeburg, Germany.

Copyright (C) 1999 Society for Neuroscience $\quad 0270-6474 / 99 / 195683-10 \$ 05.00 / 0$
}

ciencies in long-term retention of several learning tasks and markedly attenuated LTP (Bourtchuladze et al., 1994). In addition, creation of transgenic mice carrying a CRE-regulated reporter construct revealed that CRE-mediated gene expression is indeed increased in response to stimuli that generate LTP (Impey et al., 1996, 1998a).

CREB is constitutively expressed and, after phosphorylation on Ser-133, it becomes active in promoting transcription from the CRE. A number of protein kinases, including cAMP-dependent protein kinase $\mathrm{A}$ (PKA) and $\mathrm{Ca}^{2+} /$ calmodulin-dependent kinase II (CaMKII), has been implicated in CREB phosphorylation (Sheng et al., 1991; Hagiwara et al., 1993; Ginty et al., 1994; Sun et al., 1994). Thus, CREB integrates increases in cAMP and $\mathrm{Ca}^{2+}$ by inducing synergistic increases in CRE-mediated transcription.

Genetic evidence for the importance of CREB in long-term memory by far exceeds knowledge of the physiology of its activation. Progress on this front has been hampered by the difficulty of monitoring CREB activation after electrically stimulated synaptic activity or plasticity. Although we and others have detected nuclear CREB phosphorylation in response to LTP-inducing synaptic stimuli in hippocampal slices (Matthies et al., 1997b), as well as in dissociated hippocampal neurons in vitro (Bito et al., 1996; Deisseroth et al., 1996), so far activation of CREB during hippocampal LTP has not been shown in intact animals in vivo. In the present study, we addressed this question by using a phosphospecific CREB antibody for immunocy tochemistry on brain tissue from chronically implanted rats. This approach allowed us to define the spatiotemporal pattern of hippocampal CREB phosphorylation after tetanic stimulation of the perforant pathway.

\section{MATERIALS AND METHODS}

Animals. Experiments were performed with male Wistar rats [Shoe:Wist (Shoe)] from Tierzucht Schönwalde, aged 8 weeks at the beginning of the 

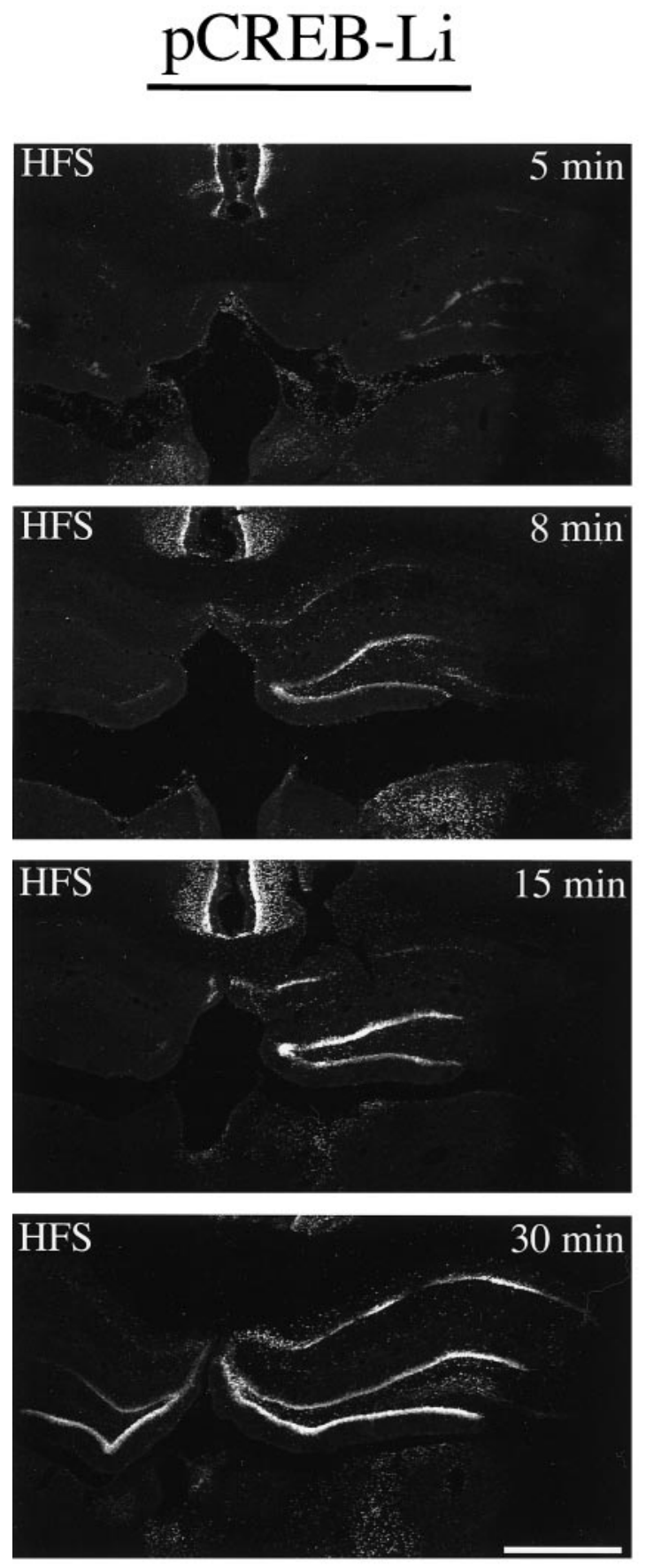

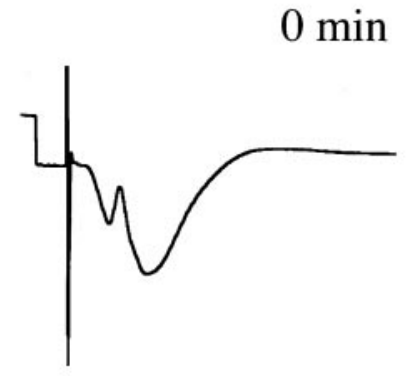

0 min

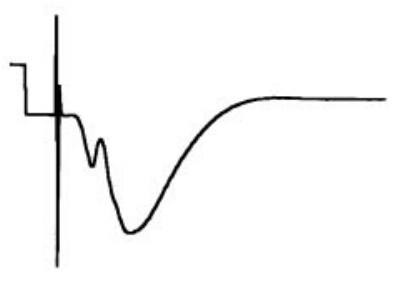

$0 \mathrm{~min}$

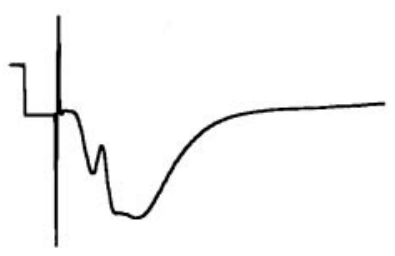

0 min

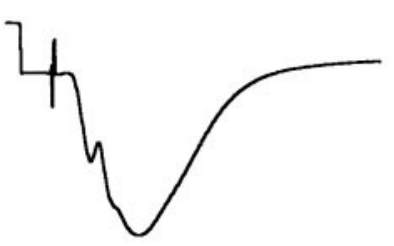

5 min

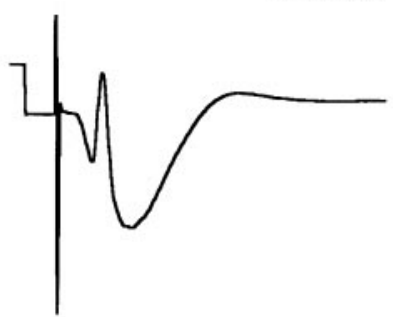

$8 \mathrm{~min}$

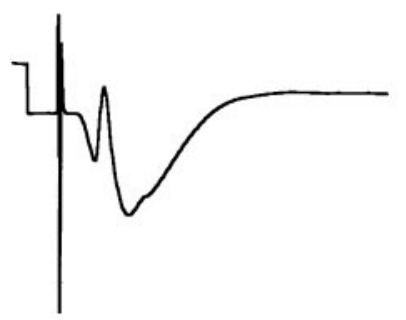

$15 \mathrm{~min}$

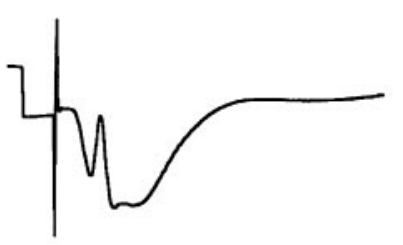

$30 \mathrm{~min}$

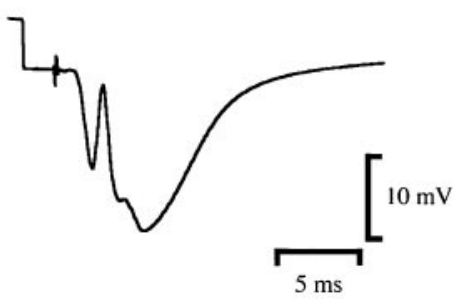

Figure 1. Rapid induction of CREB phosphorylation in hippocampal LTP. Left, Immunofluorescent confocal images of coronal hippocampal sections of animals that were subjected to high-frequency stimulation $5(n=4), 8(n=3), 15(n=4)$, or $30(n=6)$ min before vascular perfusion. Sections were immunocytochemically stained with anti-pCREB antibodies. Representative results from one of three independent experiments performed using the same method. Total number of animals used per time point is given above. Right, Corresponding electrophysiological recording before tetanization and at indicated time points after tetanization. Note that CREB phosphorylation is rapidly induced in response to stimuli that generate LTP. HFS, High-frequency stimulation. Scale bar, $1 \mathrm{~mm}$.

experiment. Animals were kept in groups of five rats per cage under controlled laboratory conditions $(12 \mathrm{hr}$ light/dark cycle, temperature $20 \pm 2^{\circ} \mathrm{C}$, humidity $55-60 \%$ ). After surgery, animals were housed individually in transparent plastic cages throughout the experiment and had access to food and water ad libitum.

Surgery. For all procedures, ethical approval was sought before the experiments according to the requirements of the German National Act on the Use of Experimental Animals. To record the monosynaptic evoked field potential and to induce LTP in the dentate gyrus after stimulation of the entorhinal-dentate pathway, animals were chronically implanted with a bipolar stimulation electrode in the ipsilateral perforant pathway (angular bundle) and a monopolar recording electrode in the dorsal blade of the ipsilateral dentate gyrus. The electrodes were made from polyurethane-coated stainless steel wires with a diameter of $125 \mu \mathrm{m}$. The animals were deeply anesthetized with pentobarbital $(40 \mathrm{mg} / \mathrm{kg}$, i.p.) and mounted on a David Kopf (Tujunga, CA) stereotaxic instrument with $\lambda$ 


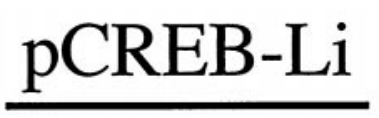

\section{pop-spike potentiation}
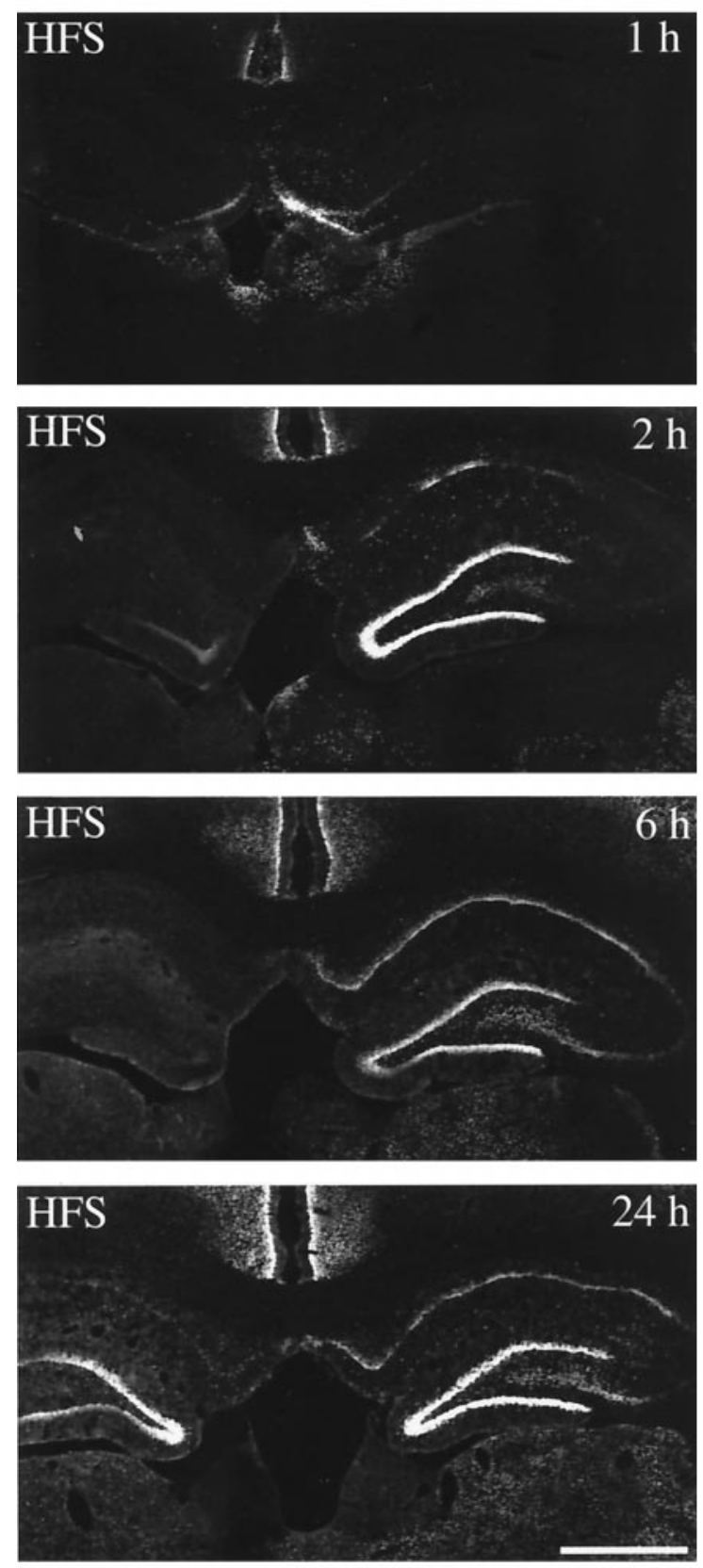

$0 \mathrm{~min}$

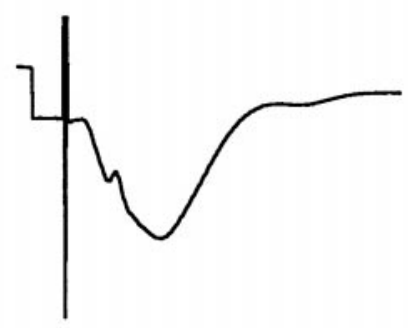

$0 \mathrm{~min}$

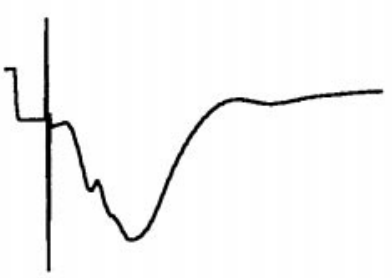

$0 \mathrm{~min}$

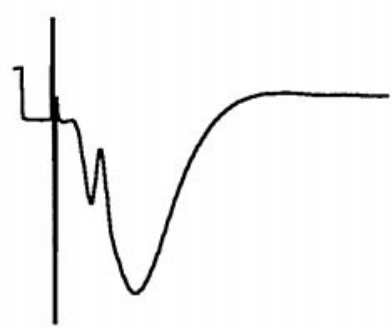

$0 \mathrm{~min}$

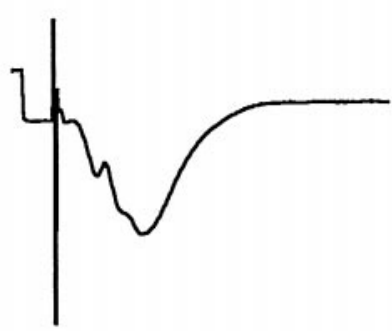

$1 \mathrm{~h}$

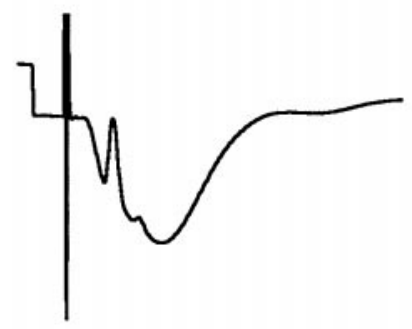

$2 \mathrm{~h}$

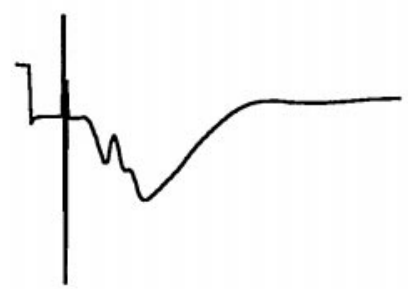

$6 \mathrm{~h}$

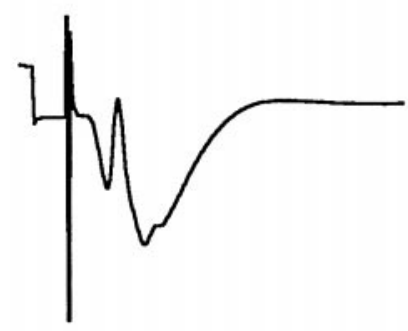

$24 \mathrm{~h}$

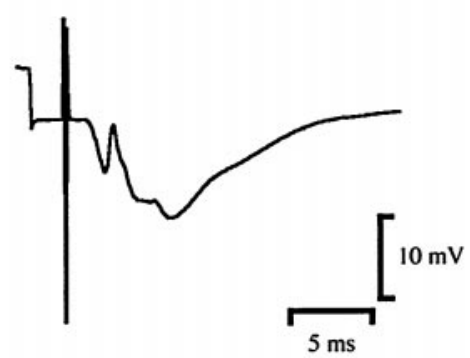

Figure 2. Second peak of CREB phosphorylation in hippocampal LTP. Left, Immunofluorescent confocal images of coronal hippocampal sections of animals that were subjected to high-frequency stimulation $1(n=6), 2(n=3), 6(n=3)$, or $24(n=3)$ hr before vascular perfusion. Sections were immunocytochemically stained with anti-pCREB antibodies. Representative results from one of three independent experiments performed using the same method. Total number of animals used per time point is given above. Right, Corresponding electrophysiological recording before tetanization and at indicated time points after tetanization. Note that CREB phosphorylation declines $1 \mathrm{hr}$ after tetanization, and a second long-lasting peak occurs from 2 to $24 \mathrm{hr}$. HFS, High-frequency stimulation. Scale bar, $1 \mathrm{~mm}$.

$1 \mathrm{~mm}$ below bregma. Small holes were drilled into the scull at the following stereotaxic coordinates: anteroposterior (AP), $-2.8 \mathrm{~mm}$; lateral (L), $1.8 \mathrm{~mm}$; and AP, $-6.8 \mathrm{~mm}$; L, $4.1 \mathrm{~mm}$ (according to Paxinos and Watson, 1982). Electrodes were placed under electrophysiological guidance. Two wires connected to miniature screws were placed into the nasal bone and served as ground and reference electrodes. All electrodes were connected to a flexible miniature socket and fixed with acrylic dental cement. Animals were allowed to recover for at least $10 \mathrm{~d}$ after surgery.
Electrophysiological recording. For electrophysiological recording, animals were connected to a model 2100 stimulator (A-M Systems, Inc., Everett, WA) and an AC-coupled amplifier (frequency range of $2 \mathrm{~Hz}$ to $10 \mathrm{kHz}$ ) through flexible, shielded cable and a swivel, thus allowing free movement. A MUSYCS interface and personal computer controlled by FAMOS software (Integrated measurement control Meßsysteme, Berlin, Germany) were used to generate trigger signals for driving the stimulator and calibration signals at the amplifier input. To elicit evoked potentials, 


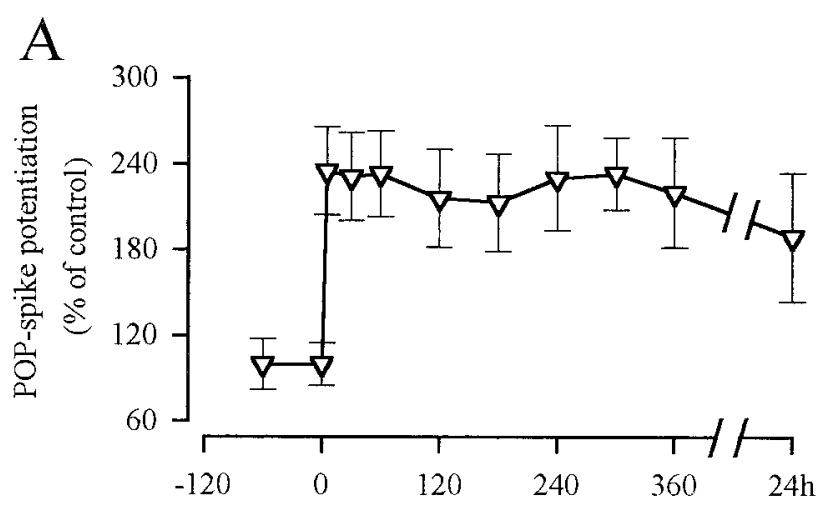

$\mathrm{B}$

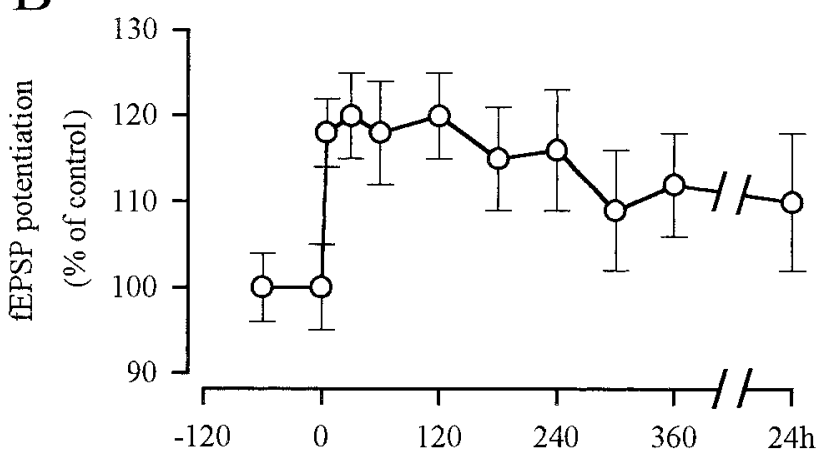

$\mathrm{C}$

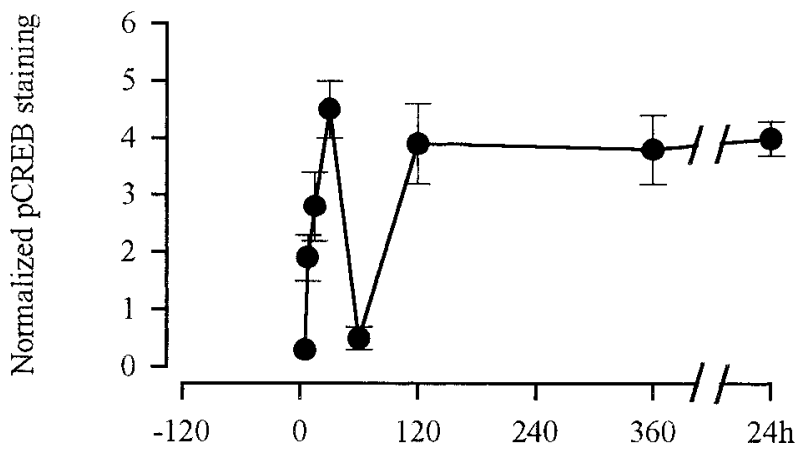

Time after tetanization ( $\mathrm{min})$

Figure 3. Biphasic increase in CREB phosphorylation during hippocampal LTP. $A$, Quantitative analysis of pop-spike potentiation in the ipsilateral dentate gyrus in response to high-frequency stimulation. $B$, Quantitative analysis of fEPSP potentiation in the ipsilateral dentate gyrus in response to high-frequency stimulation. $C$, Quantification of pCREB immunostaining in the ipsilateral dentate gyrus in response to tetanic stimulation at $0(n=3), 5(n=4), 8(n=3), 15(n=4), 30(n=6), 60$ $(n=6), 120(n=3)$, and $360(n=3)$ min and $24 \mathrm{hr}(n=3)$.

a series of eight biphasic square wave impulses with a duration of 100 $\mu \mathrm{sec} /$ half-wave was generated with a frequency of $0.2 \mathrm{~Hz}$. The field potentials were digitized with a resolution of $100 \mu \mathrm{sec}$, averaged, and stored on a hard disk. The slope function of the field EPSP (fEPSP) and population spike amplitude were automatically calculated as the steepest $400 \mu \mathrm{sec}$ segment of the initial potential upstroke and the peak to peak difference, respectively. Nondecremental LTP was induced by stimulating the ipsilateral perforant pathway with 20 trains of impulses. Each train consisted of 15 pulses having the same duration as the test pulse. The frequency within the train was $200 \mathrm{~Hz}$ for high-frequency stimulation or $0.2 \mathrm{~Hz}$ for low-frequency stimulation, and the distance between trains was $5 \mathrm{sec}$. For induction of decremental LTP, animals received only two stimulus trains. Rats were removed from the recording cages either $5 \mathrm{~min}, 8 \mathrm{~min}, 15 \mathrm{~min}, 30 \mathrm{~min}, 1 \mathrm{hr}, 2 \mathrm{hr}, 6 \mathrm{hr}$, or $24 \mathrm{hr}$ after tetanization and killed by vascular perfusion. A total of 59 animals were used, and groups of at least three to six animals were analyzed per time point and treatment condition. In addition, one group of three animals received (+)-5-methyl-10,11-dihydro-5H-dibenzo [a,d] cyclohepten-5,10imine maleate (MK801) $(0.3 \mathrm{mg} / \mathrm{kg}$, i.p.) $15 \mathrm{~min}$ before tetanization.

Immunocytochemistry. Animals were deeply anesthetized with chloralhydrate $(350 \mathrm{mg} / \mathrm{kg}$, i.p.) and transcardially perfused with Tyrode's solution, followed by a fixative containing $4 \%$ paraformaldehyde and $0.2 \%$ picric acid in $0.1 \mathrm{~m}$ phosphate buffer, $\mathrm{pH} 6.9$. Brains were rapidly dissected and post-fixed in the same fixative for $2-4 \mathrm{hr}$ at room temperature. Brains were then cryoprotected in $30 \%$ sucrose, and $40 \mu \mathrm{m}$ coronal sections were cut from the hippocampal plane near the recording site using a freezing microtome. Free-floating sections were washed in TPBS (10 mM Tris, $10 \mathrm{~mm}$ phosphate buffer, $137 \mathrm{~mm} \mathrm{NaCl}$, and $0.05 \%$ thimerosal, $\mathrm{pH}$ 7.4), placed in methanol containing $0.3 \% \mathrm{H}_{2} \mathrm{O}_{2}$ for $30 \mathrm{~min}$, and incubated in $3 \%$ normal goat serum in TPBS with $0.3 \%$ Triton X-100 for $1 \mathrm{hr}$. Subsequently, the sections were incubated with either phosphospecific anti-CREB antibody, phosphorylation state-independent antiCREB antibody or phospho-specific anti-mitogen-activated protein kinase (MAPK) antibody (all from New England Biolabs, Beverly, MA) at a dilution of 1:1000 for $72 \mathrm{hr}$. Staining of primary antibody was detected using the biotin amplification procedure as described previously (Matthies et al., 1997b; Schulz et al., 1998). Briefly, tissue sections were transferred to biotinylated goat anti-rabbit IgG (1:1000 in TPBS containing $0.3 \%$ Triton X-100 and 1\% NGS; Vector Laboratories, Burlingame, $\mathrm{CA})$ for $1.5 \mathrm{hr}$, incubated in an avidin-biotinylated horseradish peroxidase complex (ABC) solution (1:200 in TPBS plus $0.3 \%$ Triton X-100; Vector Laboratories ABC Elite kit) for $45 \mathrm{~min}$, transferred to biotinylated tyramine (BT) solution [1:1000 in TPBS plus $0.01 \% \mathrm{H}_{2} \mathrm{O}_{2}$; BT was prepared as described by Adams (1992)] for 20 min, followed by a final incubation step in streptavidine-cyanin 3.18 (1:200 in TPBS plus $0.3 \%$ Triton X-100; Amersham, Braunschweig, Germany) overnight at $4^{\circ} \mathrm{C}$. Sections were then mounted onto chrome alum gelatin-subbed glass slides, dehydrated through several concentrations of alcohol, cleared in xylol, and coverslipped with DPX. Specimens were examined using a Leica (Nussloch, Germany) TCS-NT confocal laser scanning microscope. Cyanin 3.18 was imaged with $561 \mathrm{~nm}$ excitation and $590 \mathrm{~nm}$ long-pass emission filters. Images were recorded with $2.5 \times$ or $5 \times$ objectives. Staining for serine 133 phosphorylated CREB (pCREB) was quantitated using the Leica TCS-NT software. The integrated pixel intensity was determined in the ipsilateral dentate gyrus from three adjacent sections per animal and averaged. Pixel intensity was normalized to the corresponding integrated pixel intensity in the area between stratum radiatum and stratum lacunosum.

\section{RESULTS}

\section{Time course of CREB phosphorylation during} hippocampal LTP

To monitor CREB activation, chronically implanted animals were tetanized and removed from the recording cages at different time intervals ranging from $5 \mathrm{~min}$ to $24 \mathrm{hr}$. When perfusion-fixed brain sections from these animals were immunocytochemically stained using a pCREB-specific antibody, phosphorylation of the transcription factor CREB became apparent as quickly as $5 \mathrm{~min}$ after tetanization (Figs. 1, 2). At this early time point, pCREB-like immunoreactivity ( $\mathrm{Li}$ ) was confined to the granule cell nuclei of the ipsilateral dentate gyrus. After $8 \mathrm{~min}$, the staining intensity in the dentate gyrus strongly increased. In addition, pCREB-Li was also observed in a few isolated nuclei of CA1 pyramidal cells. The intensity of this staining further increased within the next $30 \mathrm{~min}$. At 30 min after tetanization, we also observed nuclear CREB phosphorylation in the contralateral dentate gyrus. One hour after tetanization, pCREB-Li in both hippocampi almost completely disappeared. A second and more sustained peak of CREB activation was observed beginning after $2 \mathrm{hr}$ and lasting for at least $24 \mathrm{hr}$. This peak followed a very similar spatial and temporal pattern as the earlier one in that it first involved the ipsilateral dentate gyrus, then CA1 region, and at $24 \mathrm{hr}$ also the contralateraldentate gyrus. At $30 \mathrm{~min}, 2 \mathrm{hr}, 6 \mathrm{hr}$, and $24 \mathrm{hr}$, CREB 


\section{pCREB-Li}
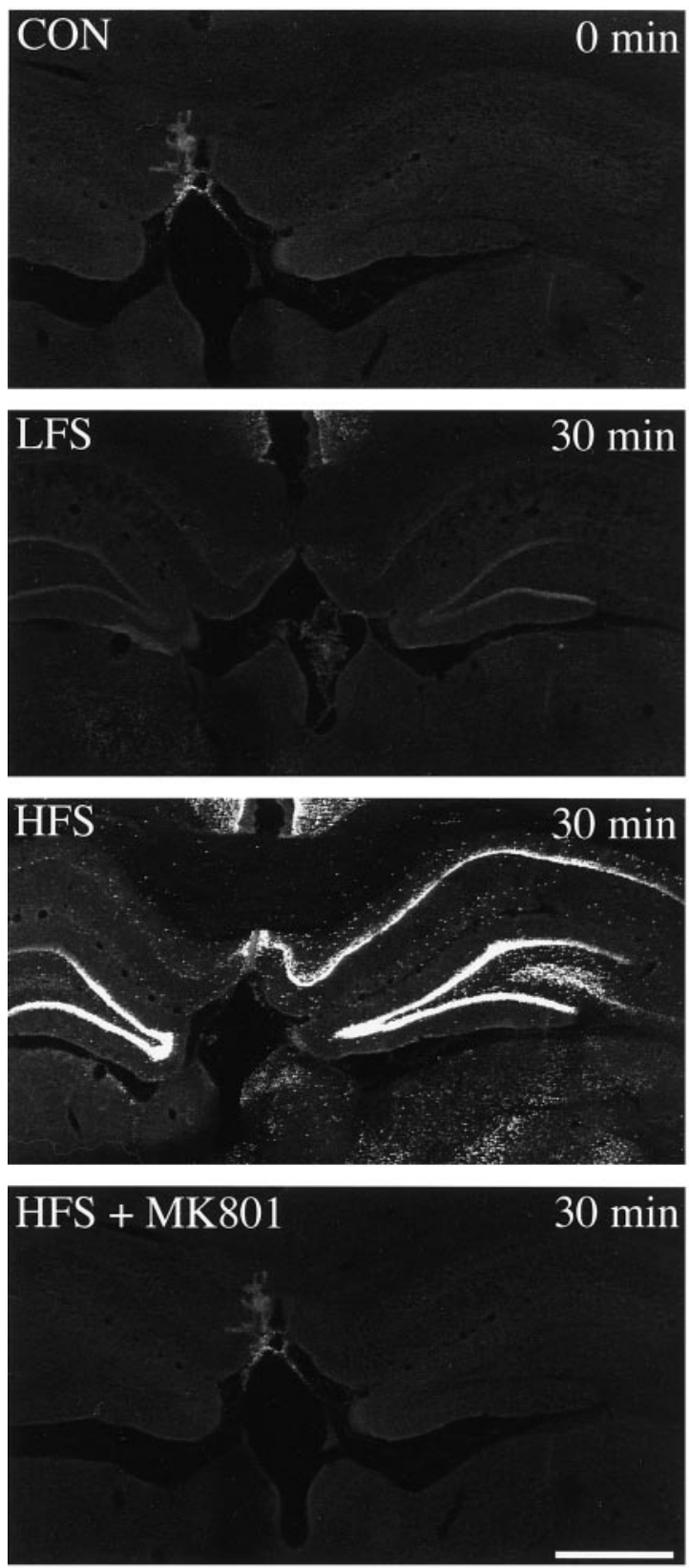

\section{pop-spike potentiation}

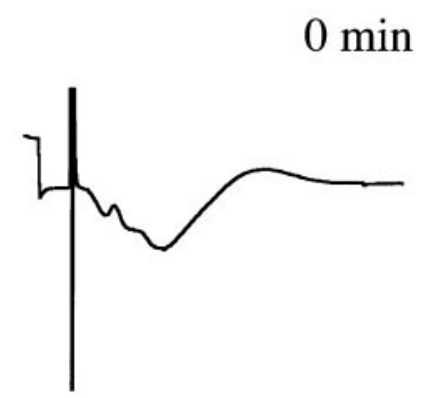

$0 \min$

$30 \mathrm{~min}$

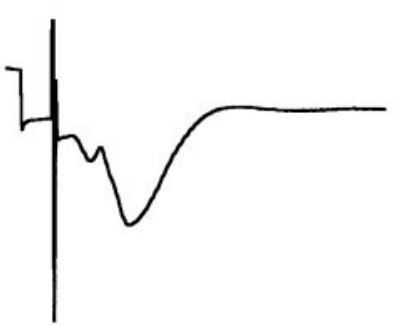

$0 \mathrm{~min}$

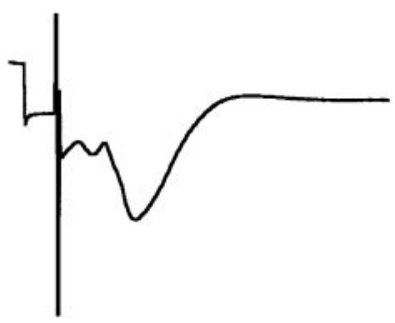

$30 \mathrm{~min}$

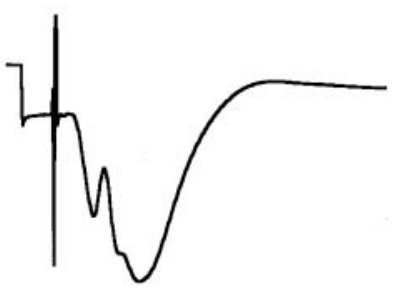

0 min

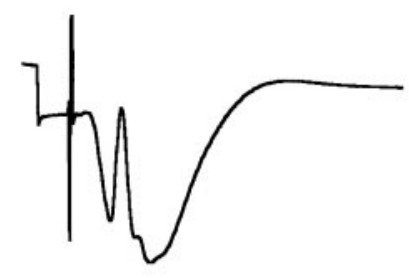

$30 \mathrm{~min}$
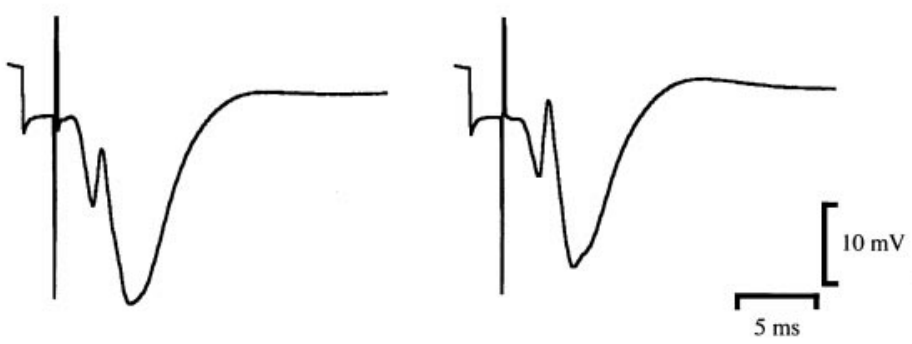

Figure 4. Stimulus specificity of hippocampal CREB activation. Left, Immunofluorescent confocal images of coronal hippocampal sections of animals that were not stimulated $(n=3)$ or subjected to either low-frequency $(n=3)$ or high-frequency $(n=3)$ stimulation 30 min before vascular perfusion. In addition, one group of animals was treated with MK801 $(0.3 \mathrm{mg} / \mathrm{kg}$, i.p.; $n=3) 15 \mathrm{~min}$ before high-frequency stimulation. Sections were immunocytochemically stained with anti-pCREB antibodies. Representative results from one of three independent experiments performed using the same method. Total number of animals used per time point is given above. Right, Corresponding electrophysiological recording before tetanization and at indicated time points after tetanization. Note that CREB phosphorylation occurs selectively in response to stimuli that generate LTP in an NMDA-dependent manner. CON, Control; LFS, low-frequency stimulation; $H F S$, high-frequency stimulation; $H F S+M K 801$, high-frequency stimulation plus MK801 treatment. Scale bar, $1 \mathrm{~mm}$.

activation was also seen in the hilar region. In contrast to the dentate gyrus and the CA1 region, nuclear CREB phosphorylation in response to high-frequency stimulation was not observed in the $\mathrm{CA} 3$ region during the various time points studied. Figure $3 C$ depicts the quantification of pCREB-Li in the ipsilateral dentate gyrus of all animals examined, clearly demonstrating the biphasic manner of pCREB. In contrast, population spike (popspike) amplitude, as well as fEPSP, were continuously enhanced during the same time period, clearly showing that LTP actually occurred in these experiments (Fig. $3 A, B$ ). 

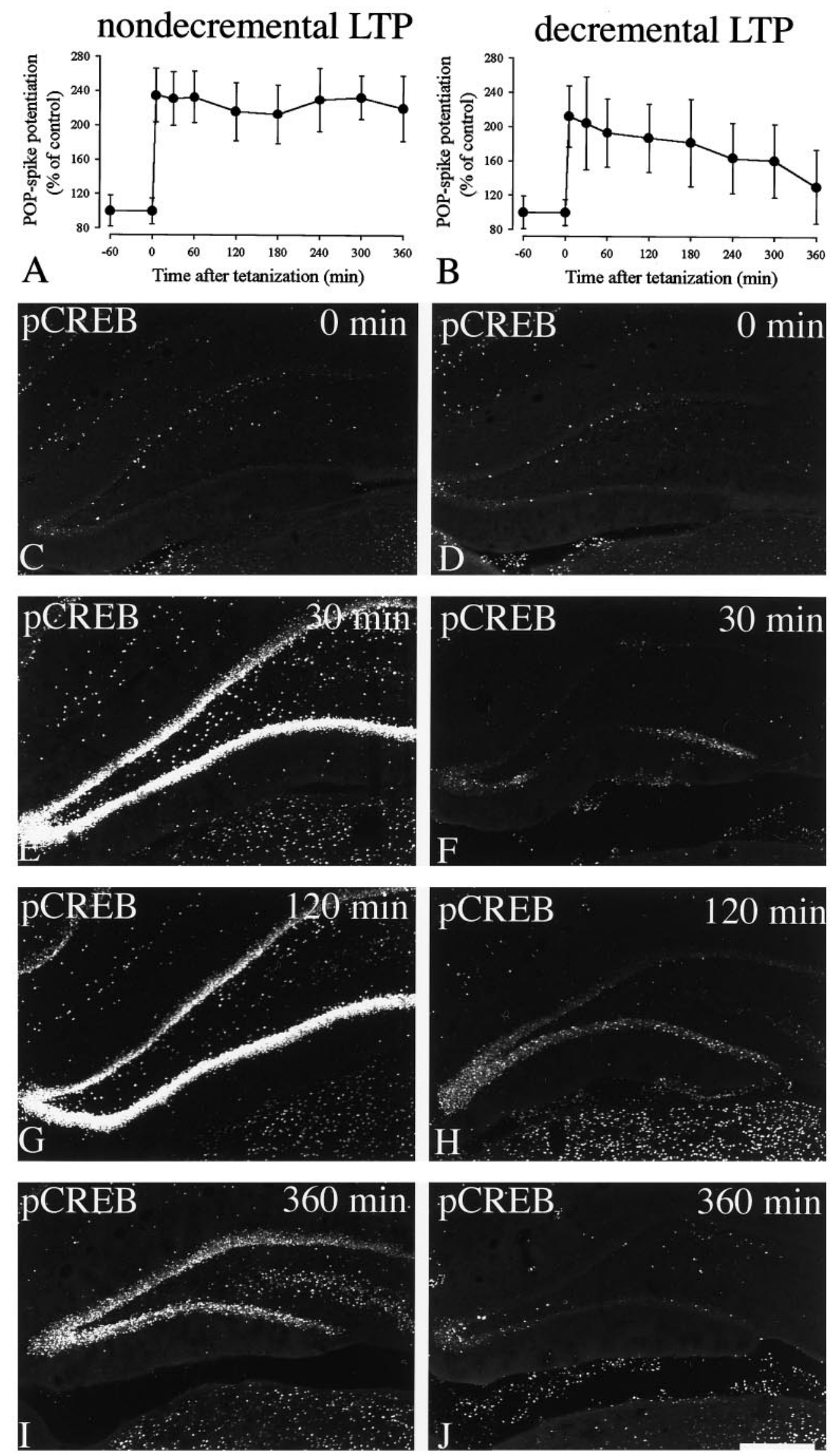


\section{Stimulus-specificity of hippocampal CREB activation}

If CREB is specifically involved in hippocampal plasticity, it should be selectively activated in response to stimuli that induce a long-lasting enhancement of synaptic strength. Therefore, in a separate series of experiments, rats were subjected to either highor low-frequency stimulation conditions known to either generate or not generate hippocampal LTP, respectively. Again, a robust induction of pCREB-Li was observed in the dentate gyrus, as well as the CA1 region, $30 \mathrm{~min}$ after high-frequency stimulation. In contrast, virtually no pCREB-Li was seen in animals that were either subjected to low-frequency stimulation or not stimulated (Fig. 4). Moreover, treatment of animals with the NMDA receptor antagonist MK801 not only prevented the induction of hippocampal LTP but also blocked the enhancement of pCREB-Li in response to high-frequency stimulation (Fig. 4).

\section{CREB is hyperphosphorylated during nondecremental, but not decremental, LTP}

Genetic evidence suggests that CREB may play a crucial role for generation of a late-phase of LTP, as well as consolidation of long-term memory. Thus, experiments were conducted to determine how tightly CREB phosphorylation correlates with nondecremental and decremental LTP. When animals received a strong stimulus (20 trains) that generated nondecremental LTP, a robust and sustained hyperphosphorylation of CREB was seen in the ipsilateral dentate gyrus (Fig. 5, left panels). This increase in CREB phosphorylation lasted for at least $6 \mathrm{hr}$. In contrast, animals that received a weak stimulus (2 trains) resulting in decremental LTP as evidenced by the decline of both pop-spike and fEPSP potentiation showed, if at all, a small and transient rise in hippocampal CREB phosphorylation within $2 \mathrm{hr}$ after tetanus, and after $6 \mathrm{hr}$, none of the animals showed any significant CREB phosphorylation (Fig. 5, right panels).

\section{Comparison between CREB phosphorylation and MAPK activation}

It has been shown recently that sequential activation of the extracellular signal-regulated kinase (ERK)/MAPK pathway and the CREB kinase ribosomal S6 kinase 2 (RSK2) may lead to phosphorylation and transactivation of CREB (Impey et al., 1998b). The activation state of MAP kinase can easily be monitored using immunocytochemistry with tyrosine 204 phosphorylation-specific MAPK antibodies. If the ERK/MAPK cascade would contribute to CREB phosphorylation, then activation of MAP kinase and CREB would be expected to follow closely overlapping patterns. However, when animals were perfused $30 \mathrm{~min}$ after tetanization and adjacent brain sections were stained with either anti-pCREB or anti-pMAPK antibodies, we found that pCREB-Li and pMAPK-Li showed strikingly different regional and subcellular distributions (Fig. $6 A-D$ ). Like pCREB$\mathrm{Li}$, pMAPK-Li was prominent in the ipsilateral dentate gyrus.
Unlike pCREB-Li, pMAPK-Li was not seen in granule cell nuclei but rather was confined to fibers and processes in the polymorph layer and hilus. In the Ammon's horn of the hippocampus, pMAPK-Li was not restricted to the CA1 region but involved also the CA3 region. In contrast to pCREB-Li, pMAPK-Li was virtually absent from pyramidal cell nuclei but was prominent in the stratum oriens, as well as stratum radiatum.

In addition, we compared the distribution of activated CREB proteins detected using a Ser-133 phosphorylation-specific antibody and total CREB proteins detected using a phosphorylation state-independent anti-CREB antibody. As shown in Figure 6, $E$ and $F$, similar levels of total immunoreactive CREB proteins were detected in all regions of both hippocampi and adjacent brain regions, indicating that the pCREB-Li staining pattern clearly reflects a response to tetanic stimulation.

\section{DISCUSSION}

In the present work, we have resolved the spatiotemporal dynamics of CREB serine 133 phosphorylation during hippocampal LTP in freely moving rats. CREB phosphorylation occurs in a biphasic manner, with a first short-lasting peak at $30 \mathrm{~min}$ and a second more sustained peak beginning after $2 \mathrm{hr}$ and lasting for at least $24 \mathrm{hr}$ (Fig. 4). CREB was selectively recruited in the dentate gyrus and the CA1, but not the CA3, region of the ipsilateral hippocampus. CREB phosphorylation was triggered in a stimulus-specific manner. Only stimuli that induce a persistent enhancement of synaptic strength (high-frequency stimulation) also produced robust increases in hippocampal pCREB-Li. Stimuli that did not induce pop-spike or fEPSP potentiation (lowfrequency stimulation) also failed to trigger phosphorylation of CREB. In fact, sustained CREB activation appears to be required for maintenance of a late-phase of LTP. Only stimuli that generated nondecremental LTP also promoted strong and long-lasting CREB phosphorylation but not stimuli that induced decremental LTP. The initial recruitment of CREB appears to depend on calcium influx via the NMDA receptor because pretreatment of animals with MK801 completely blocked the induction of hippocampal LTP and associated increases in CREB phosphorylation at the 30 min time point.

Although our in vivo data strongly support the hypothesis that CREB is an important component of a general switch that converts short-term into long-term synaptic plasticity, it is not known by which signaling cascades CREB phosphorylation is mediated. The surrounding sequences of serine 133 in mammalian CREB contain recognition sites for PKA, CaMKII, and RSK2 (Sheng et al., 1991; Hagiwara et al., 1993; Impey et al., 1998b). LTP is not a unitary phenomenon but consists of several phases (Matthies et al., 1990). An early phase of LTP that is blocked by inhibitors of CaMKII can be dissociated from late LTP, which is sensitive to PKA inhibitors (Ito et al., 1991; Frey et al., 1993; Matthies and Reymann, 1993; Huang et al., 1994). It is thus very tempting to

\section{$\leftarrow$}

Figure 5. Differential CREB phosphorylation during nondecremental and decremental LTP. Left, Nondecremental LTP was induced by stimulating the ipsilateral perforant pathway with 20 stimulus trains as described in Materials and Methods. Animals were removed from the recording cages after 0 ( $n=$ $3), 30(n=6), 120(n=3)$, or $360(n=3)$ min and subjected to vascular perfusion. Right, Decremental LTP was induced by stimulating the ipsilateral perforant pathway with two stimulus trains as described in Materials and Methods. Animals were removed from the recording cages after $0(n=3), 30$ $(n=3), 120(n=3)$, or $360(n=3)$ min and subjected to vascular perfusion. Sections were immunocytochemically stained with anti-pCREB antibodies. $A, B$, Quantitative analysis of pop-spike potentiation in the ipsilateral dentate gyrus. $C-J$, Immunofluorescent confocal images of coronal hippocampal sections. Representative results from one of three independent experiments performed using the same method. Total number of animals used per time point is given above. Note that only stimuli that generate nondecremental LTP induced sustained CREB phosphorylation but not stimuli that generate decremental LTP. Scale bar, $1 \mathrm{~mm}$. 

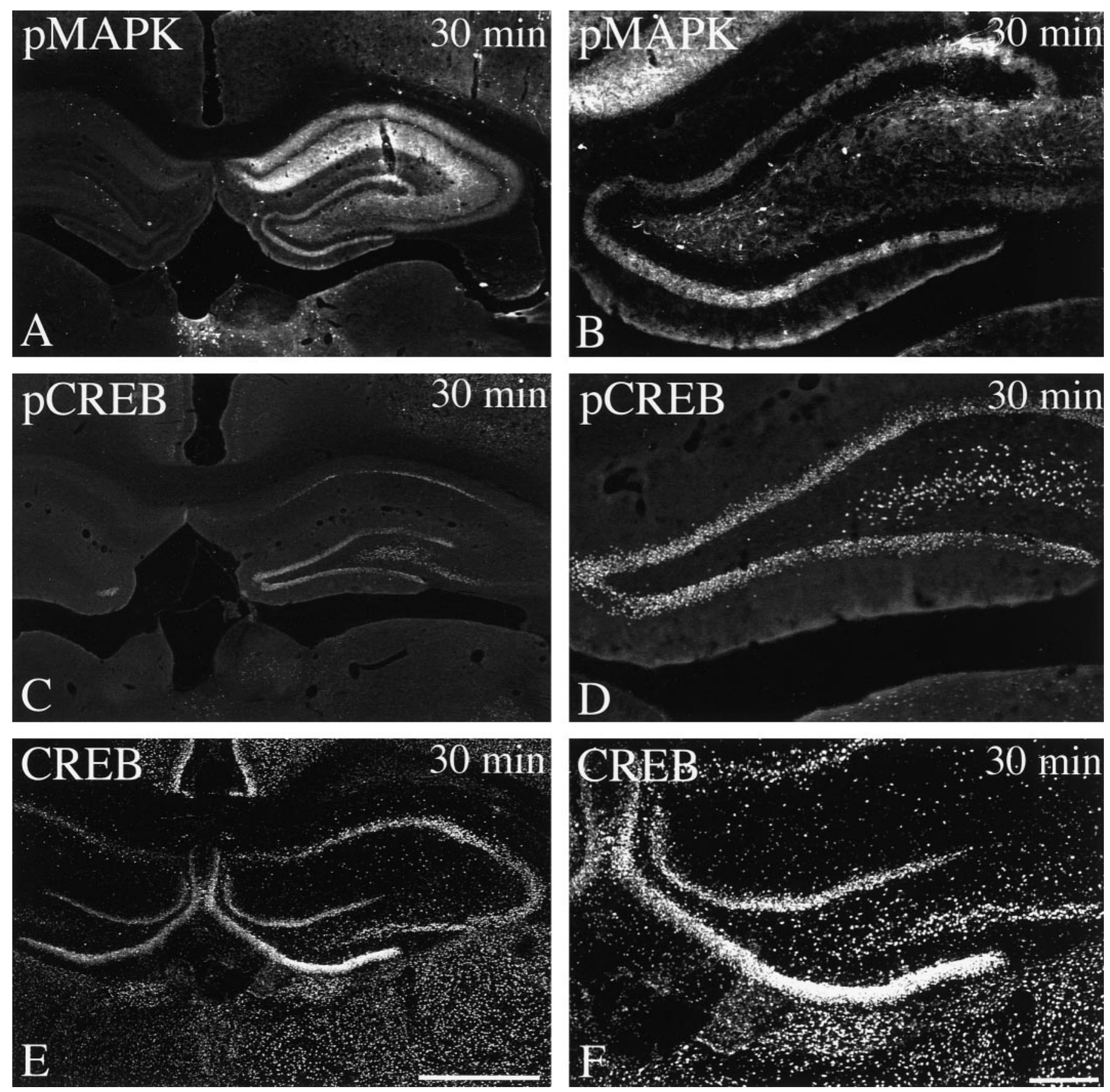

Figure 6. Comparison of pMAPK- and pCREB-like immunoreactivities at $30 \mathrm{~min}$ after tetanus. Immunofluorescent confocal images of coronal hippocampal sections of animals that were subjected to high-frequency stimulation $30 \mathrm{~min}(n=3)$ before vascular perfusion. Sections were immunocytochemically stained with either anti-pMAPK, anti-pCREB, or anti-CREB antibodies. Representative results from one of three independent experiments performed using the same method. Note that both MAPK and CREB are clearly activated in the ipsilateral dentate gyrus but show strikingly different distributions $30 \mathrm{~min}$ after tetanization. Similar levels of total immunoreactive CREB proteins were detected in all regions of both hippocampi and adjacent brain regions, indicating that the pCREB-Li staining pattern reflects a response to tetanic stimulation. Scale bars: (in $E$ ) $A, C, E, 1$ mm; (in $F) B, D, F, 1 \mathrm{~mm}$.

speculate that CaMKII may be involved in the initial peak of CREB phosphorylation, whereas PKA may primarily contribute to the second more sustained peak of CREB phosphorylation. Recent work by Impey et al. (1998b) suggests that sequential activation of the ERK/MAPK pathway and the CREB kinase RSK2 may lead to phosphorylation and transactivation of CREB. In the present study, we monitored the activation state of the MAPK cascade using immunocytochemistry with tyrosine 204 phosphorylation-specific MAPK antibodies and observed strikingly different patterns of CREB phosphorylation and MAPK activation. Whereas 30 min post-tetanus pCREB-Li was clearly confined to the nuclei of granule and CA1 pyramidal cells, pMAPK-Li was prominent in fibers and processes of these cells. These findings would suggest a model in that synaptic activation of MAPK would trigger nuclear translocation of RSK2, which in turn phosphorylates CREB. Alternatively, MAPK may serve 
other functions during hippocampal LTP, i.e., phosphorylation of neural cell adhesion molecules (Bailey et al., 1997).

In freely moving animals, tetanic stimulation of the perforant path projection is known to elicit long-term potentiation in the ipsilateral dentate gyrus. Although CREB phosphorylation was first noted in the ipsilateral dentate gyrus, it was not limited there. pCREB-Li was also seen in the ipsilateral CA1 region (but not CA3), as well as the contralateral dentate gyrus. The simplest explanation for this finding would be that information about enhanced synaptic activity is relayed via intrahippocampal circuits from the ipsilateral dentate gyrus to the ipsilateral CA1 region, as well as to the contralateral dentate gyrus. Alternatively, the entorhinal cortex may directly project to the contralateral dentate gyrus (Amaral and Witter, 1989; Berger et al., 1997). Indeed, we have found previously that, after tetanic stimulation of the perforant path projection, a delayed form of long-term potentiation can be recorded from the contralateral dentate gyrus (M. Krug, unpublished observation). Excitation of the ipsilateral CA1 region would be expected to occur via the "classical" trisynaptic loop involving mossy fibers terminating at CA3 pyramidal cells and the Schaffer collateral-commissural pathway. However, significant CREB phosphorylation was not seen in CA3 pyramidal cell nuclei. This is perhaps not surprising because the form of long-term potentiation found at mossy fiber synapses is independent of NMDA receptors and requires a rise in presynaptic $\mathrm{Ca}^{2+}$ (Collingridge and Bliss, 1995; Nicoll and Malenka, 1995). Here, we show that CREB phosphorylation is critically dependent on $\mathrm{Ca}^{2+}$-influx via postsynaptic NMDA receptors. Alternatively, a direct activation of CA1 pyramidal cells from the entorhinal cortex seems possible (Amaral and Witter, 1989; Berger et al., 1997).

Although substantial genetic evidence for a role of CREB in memory formation exists and the present study clearly demonstrates that CREB is specifically recruited during hippocampal LTP in vivo, it is not known which of the many CREB/activating transcription factor (ATF) or cAMP responsive element-binding modulator (CREM) protein isoforms are involved in long-term neuronal plasticity. Here, we selectively monitored CREB serine 133 phosphorylation showing that $\mathrm{CREB}$ is recruited at two distinct time points. Whereas the first peak of CREB phosphorylation appears to be rapidly terminated by dephosphorylation, the second peak of CREB phosphorylation appears to be more sustained and lasts for at least $24 \mathrm{hr}$. Interestingly, CRE-mediated gene expression can be detected as early as $2 \mathrm{hr}$ after tetanus and reaches a maximum at $4-6 \mathrm{hr}$, suggesting that sustained CREB phosphorylation is required for induction of persistent CREmediated gene expression. However, it remains open to question what role inhibitory CREM isoforms may play in the termination of the second peak of CREB phosphorylation.

It is important to note that interesting correlations exist between the time course of CREB phosphorylation and the expression of immediate-early genes after induction of LTP. In awake animals, tetanic stimulation of the perforant path resulted in a rapid induction of Fos-related antigen but not of Fos or Fos-B proteins, which may provide a link between CREB activation and nuclear gene expression (for review, see Hughes and Dragunow, 1995). Subsequently, 1-6 hr after LTP induction, a number of genes appear to be upregulated (Hevroni et al., 1998). Moreover, induction of long-term facilitation in Aplysia has been shown to be associated with multiple waves of protein synthesis. Here, a group of 10 proteins is rapidly induced within the first hour after repeated exposure to 5-HT. These early changes are followed by a second wave of changes in other proteins at $3 \mathrm{hr}$, and later by a third more persistent wave of protein synthesis (Barzilai et al., 1989; Abel and Kandel, 1998). Similarly, a biphasic time course of protein synthesis has also been observed in simple learning paradigms (for review, see Matthies, 1989). It is thus conceivable that the biphasic phosphorylation of CREB may participate in triggering multiple waves of protein synthesis required for a plastic change in synaptic efficacy.

In summary, the spatial and temporal pattern of CREB phosphorylation during hippocampal LTP in vivo is more complex than previously expected. The transcription factor CREB is specifically recruited at two distinct time points in response to stimuli that generate a persistent enhancement of synaptic efficacy. These findings strongly reinforce the notion that the CREB/ATF family of transcription factors is an important component of a molecular switch that converts short-term into long-term synaptic plasticity.

\section{REFERENCES}

Abel T, Kandel ER (1998) Positive and negative regulatory mechanisms that mediate long-term memory storage. Brain Res Rev 26:360-378.

Adams JC (1992) Biotin amplification of biotin and horseradish peroxidase signals in histochemical stains. J Histochem Cytochem 40:1457-1463.

Amaral DG, Witter MP (1989) The three-dimensional organization of the hippocampal formation: a review of anatomical data. Neuroscience 31:571-591.

Bailey CH, Bartsch D, Kandel ER (1996) Towards a molecular definition of long-term memory storage. Proc Natl Acad Sci USA 93:13445-13452.

Bailey CH, Kaang BK, Chen M, Martin KC, Lim CS, Casadio A, Kandel ER (1997) Mutation in the phosphorylation sites of MAP kinase blocks learning-related internalization of apCAM in Aplysia sensory neurons. Neuron 18:913-924.

Bartsch D, Ghirardi M, Skehel PA, Karl KA, Herder SP, Chen M, Bailey CH, Kandel ER (1995) Aplysia CREB2 represses long-term facilitation: relief of repression converts transient facilitation into long-term functional and structural change. Cell 83:979-992.

Bartsch D, Casadio A, Karl KA, Serodio P, Kandel ER (1998) CREB1 encodes a nuclear activator, a repressor, and a cytoplasmic modulator that form a regulatory unit critical for long-term facilitation. Cell 95:211-223.

Barzilai A, Kennedy TE, Sweatt JD, Kandel ER (1989) 5-HT modulates protein synthesis and the expression of specific proteins during longterm facilitation in Aplysia sensory neurons. Neuron 2:1577-1586.

Berger TW, Yeckel MF, Thiels E (1997) Network determinants of hippocampal synaptic plasticity. In: Long-term potentiation, Vol 3 (Baudry M, Davis JL, eds), pp 233-267. Cambridge, MA: MIT.

Bito H, Deisseroth K, Tsien RW (1996) CREB phosphorylation and dephosphorylation: a $\mathrm{Ca}^{2+}$ - and stimulus duration-dependent switch for hippocampal gene expression. Cell 87:1203-1214.

Bliss TVP, Collingridge GL (1993) A synaptic model of memory: longterm potentiation in the hippocampus. Nature 361:31-39.

Bourtchuladze R, Frenguelli B, Blendy J, Cioffy D, Schutz G, Silva AJ (1994) Deficient long-term memory in mice with a targeted mutation of the cAMP-responsive element binding protein. Cell 79:59-68.

Collingridge GL, Bliss TVP (1995) Memories of NMDA receptors and LTP. Trends Neurosci 18:54-56.

Dash PK, Hochner B, Kandel ER (1990) Injection of the cAMPresponsive element into the nucleus of Aplysia sensory neurons blocks long-term facilitation. Nature 354:718-721.

Deisseroth K, Bito H, Tsien RW (1996) Signaling from synapse to nucleus: postsynaptic CREB phosphorylation during multiple forms of hippocampal synaptic plasticity. Neuron 16:89-101.

Frank DA, Greenberg ME (1994) CREB: a mediator of long-term memory from mollusks to mammals. Cell 79:5-8.

Frey U, Huang YY, Kandel ER (1993) Effects of cAMP stimulate a late stage of LTP in hippocampal CA1 neurons. Science 260:1661-1664.

Ginty DD, Bonni A, Greenberg ME (1994) Nerve growth factor activates a Ras-dependent protein kinase that stimulates c-fos transcription via phosphorylation of CREB. Cell 77:713-725. 
Hagiwara M, Brindle P, Harootunian A, Armstrong R, Rivier J, Vale W, Tsien R, Montminy MR (1993) Coupling of hormonal stimulation and transcription via the cyclic AMP-responsive factor CREB is rate limited by nuclear entry of protein kinase A. Mol Cell Biol 13:4852-4859.

Hevroni D, Rattner A, Bundman M, Lederfein D, Gabarah A, Mangelus M, Silverman MA, Kedar H, Naor C, Kornuc M, Hanoch T, Seger R, Theill LE, Nedivi E, Richter-Levin G, Citri Y (1998) Hippocampal plasticity involves extensive gene induction and multiple cellular mechanisms. J Mol Neurosci 10:75-98.

Huang YY, Kandel ER (1994) Recruitment of long-lasting and protein kinase A-dependent long-term potentiation in the CA1 region of the hippocampus requires repeated tetanization. Learn Mem 1:74-82.

Hughes P, Dragunow M (1995) Induction of immediate-early genes and the control of neurotransmitter-regulated gene expression within the nervous system. Pharmacol Rev 47:133-178.

Impey S, Mark M, Villacres EC, Chavkin C, Storm DR (1996) Induction of CRE-mediated gene expression by stimuli that generate long-lasting LTP in area CA1 of the hippocampus. Neuron 16:973-982.

Impey S, Smith DM, Obrietan K, Donahue R, Wade C, Storm DR (1998a) Stimulation of cAMP response element (CRE)-mediated transcription during contextual learning. Nat Neurosci 1:595-601.

Impey S, Obrietan K, Wong ST, Poser S, Yano S, Wayman G, Deloume JC, Chan G, Storm DR (1998b) Cross talk between ERK and PKA is required for $\mathrm{Ca}^{2+}$ stimulation of CREB-dependent transcription and ERK nuclear translocation. Neuron 21:869-883.

Ito I, Hidaka H, Sugiama H (1991) Effects of KN-62, a specific inhibitor of calcium/calmodulin-dependent protein kinase II, on long-term potentiation in the rat hippocampus. Neurosci Lett 121:119-121.

Krug M, Lössner B, Ott T (1984) Anisomycin blocks the late phase of long-term potentiation in the dentate gyrus of freely moving rats. Brain Res Bull 13:39-42.

Matthies H (1989) In search of cellular mechanisms of memory. Prog Neurobiol 32:277-349.

Matthies H, Frey U, Reymann K, Krug M, Jork R, Schroeder H (1990) Multiple mechanisms and multiple stages of LTP. In: Excitatory amino acids and neuronal plasticity (Ben Ari Y, ed), pp 359-368. New York: Plenum.

Matthies Jr H, Reymann KG (1993) Protein kinase A inhibitors prevent the maintenance of hippocampal long-term potentiation. NeuroReport 4:712-714.

Matthies H, Schulz S, Höllt V, Krug M (1997a) Inhibition by compactin demonstrates a requirement of isoprenoid intermediates in hippocampal LTP. Neuroscience 79:341-346.

Matthies H, Schulz S, Thiemann W, Siemer H, Schmidt H, Krug M, Höllt V (1997b) Design of a multiple slice interface chamber and application for resolving the temporal pattern of CREB phosphorylation in hippocampal long-term potentiation. J Neurosci Methods 78:173-179.

Nicoll RA, Malenka RC (1995) Contrasting properties of two forms of long-term potentiation in the hippocampus. Nature 377:115-117.

Otani S, Ben Ari Y (1993) Biochemical correlates of long-term potentiation in hippocampal synapses. Int Rev Neurobiol 35:1-41.

Paxinos G, Watson C (1983) The rat brain in stereotaxic coordinates. New York: Academic.

Schulz S, Höllt V (1998) Opioid withdrawal activates MAP kinase in locus coeruleus neurons in morphine-dependent rats in vivo. Eur J Neurosci 10:1196-1201.

Sheng M, Thompson MA, Greenberg ME (1991) CREB: a $\mathrm{Ca}^{2+}$ regulated transcription factor phosphorylated by calmodulindependent kinases. Science 252:427-430.

Stevens CF (1994) CREB and memory consolidation. Neuron 13:769-770.

Sun P, Enslen H, Myung PS, Maurer RA (1994) Differential activation of CREB by $\mathrm{Ca}^{2+} /$ calmodulin-dependent protein kinases type II and IV involves phosphorylation of a site that negatively regulates activity. Genes Dev 8:2527-2539.

Yin JC, Wallach JS, Del Vecchio M, Wilder EL, Zhou H, Quinn WG, Tully $\mathrm{T}$ (1994) Induction of a dominant negative CREB transgene specifically blocks long-term memory in Drosophila. Cell 79:49-58.

Yin JC, Del Vecchio M, Zhou H, Tully T (1995) CREB as a memory modulator: induced expression of a dCREB2 activator isoform enhances long-term memory in Drosophila. Cell 81:107-115. 